\title{
Structural Basis of Template Boundary Definition in Tetrahymena Telomerase
}

\author{
Linnea I. Jansson ${ }^{1}$, Ben M. Akiyama ${ }^{2}$, Alexandra Ooms ${ }^{1}$, Cheng Lu ${ }^{1}$, Seth M. Rubin ${ }^{1}$, and \\ Michael D. Stone ${ }^{1}$ \\ ${ }^{1}$ Department of Chemistry and Biochemistry, University of California Santa Cruz, Santa Cruz, \\ California, USA \\ ${ }^{2}$ Department of Chemistry and Molecular Genetics, University of Colorado School of Medicine, \\ Denver, Colorado, USA
}

\begin{abstract}
Telomerase is required to maintain repetitive G-rich telomeric DNA sequences at chromosome ends. To do so, the telomerase reverse transcriptase (TERT) subunit reiteratively uses a small region of the integral telomerase RNA (TER) as a template. An essential feature of telomerase catalysis is the strict definition of the template boundary to determine the precise TER nucleotides to be reverse transcribed by TERT. We report the $3 \AA$ crystal structure of the Tetrahymena TERT RNA binding domain (tTRBD) bound to the template boundary element (TBE) of TER. tTRBD is wedged into the base of the TBE RNA stem-loop and each of the flanking RNA strands wraps around opposite sides of the protein domain. The structure illustrates how the tTRBD establishes the template boundary by positioning the TBE the correct distance from the TERT active site to prohibit copying of non-template nucleotides.
\end{abstract}

\section{INTRODUCTION}

Telomeres are essential nucleoprotein structures that help maintain genome integrity by differentiating natural chromosome ends from sites of DNA damage ${ }^{1}$. In most cells, a progressive shortening of telomere length with each round of cell division provides a molecular signal for cell aging and regulates entry into permanent cell growth arrest or apoptosis ${ }^{2}$. In contrast, cells possessing a high level of proliferative capacity (i.e. stem cells, inflammatory cells, and other self-renewing tissues) maintain telomere length through the enzymatic action of the specialized reverse transcriptase, telomerase ${ }^{3}$. The discovery of lossof-function telomerase mutations in patients with diseases of the hematopoietic system (ie.

\footnotetext{
Users may view, print, copy, and download text and data-mine the content in such documents, for the purposes of academic research, subject always to the full Conditions of use:http://www.nature.com/authors/editorial_policies/license.html\#terms

Correspondence should be addressed to M.D.S. (mds@ucsc.edu).

Accession codes.

Coordinates and structure factors have been deposited in the Protein Data Bank under accession code 5C9H.

Author Contributions

L.I.J., B.M.A, A.O., C.L., S.M.R., and M.D.S designed the experiments. L.I.J., B.M.A, S.M.R., and M.D.S. wrote the manuscript.

Competing Financial Interests

The authors declare no competing financial interests.
} 
Dyskeratosis Congenita and Aplastic Anemia) illustrates the requirement for telomerase in highly proliferative tissues ${ }^{4}$. On the other hand, aberrant activation of telomerase is deleterious, providing a mechanism for $\sim 90 \%$ of human cancers to bypass the tumor suppressing activity of telomere shortening 5 .

The minimal requirements for in vitro reconstitution of the active telomerase ribonucleoprotein (RNP) include a telomerase RNA (TER) and the telomerase reverse transcriptase (TERT) protein (Fig. 1a,b) ${ }^{6}$.The telomerase RNP directs de novo DNA synthesis at chromosome 3' ends utilizing a unique reverse transcription mechanism wherein an internal region of the telomerase RNA serves as the template (Fig. 1c) ${ }^{7}$ During telomere repeat synthesis, telomerase binds a single-stranded DNA substrate via Watson-Crick basepairing with the RNA template and through additional TERT-DNA interactions termed 'anchor sites' ${ }^{8-11}$. Next, TERT catalyzes the synthesis of a telomere DNA repeat according to the sequence specified by the RNA template. Completion of a telomere repeat initiates telomerase RNP translocation to reposition the TERT active site, telomerase RNA, and the DNA substrate to the original DNA primer alignment configuration to allow for further rounds of repeat addition ${ }^{12,13}$.The precise definition of a region of TER that may access the TERT active site and template the synthesis of a telomere DNA repeat is a hallmark of telomerase function; however, the precise structural details of how this template boundary is defined have not been characterized.

TERTs from diverse organisms are highly conserved and share a common domain organization including: the essential N-terminal domain (TEN), RNA binding domain (RBD), reverse transcriptase domain (RT), and C-terminal extension (CTE) (Fig. 1a). The contribution of individual TERT domains to telomerase assembly and catalytic activity has been studied using in vitro deletion and mutagenesis studies, as well as single molecule FRET. The TEN domain makes contacts with both DNA and RNA and is essential for telomerase processivity ${ }^{10,14-16}$. RBD binds TER using several conserved regions known as the T-motif, CP-motif, and ciliate-specific CP2-motif (Supplementary Fig.1 and Supplementary Fig.2) ${ }^{17,18}$. TERs are far more divergent with respect to both size and sequence, but maintain a conserved organization of multiple RNA elements including: a template boundary element (TBE), the template region, an RNA pseudoknot domain, and a distal RNA stem-loop (Fig. 1B) ${ }^{19}$. In telomerase from the model organism Tetrahymena thermophila, the base of stem-loop II and its flanking single-stranded RNA comprise the TBE of TER, and is proposed to interact with the T-, CP-, and CP2-motifs within the TERT$\mathrm{RBD}^{20,21}$. Consistent with this notion, sequence mutations within the TBE or each of the conserved TERT-RBD motifs result in a loss of protein-RNA binding affinity, as well as template read-through defects when assayed in direct primer extension assays in vitro ${ }^{17,18,20-22}$.

The high-resolution structure of the Tetrahymena TERT-RBD (tTRBD) domain revealed the organization of the T- and CP-motifs, which together form a putative RNA binding pocket ${ }^{23}$. However, the tTRBD structure did not include RNA and lacked the CP2-motif, which is essential for high affinity RNA interactions ${ }^{21}$. The more recently reported structures of TRBDs from diverse organisms indicate that this domain shares a common overall folding topology ${ }^{24-26}$. Moreover, the structure of the Tribolium castaneum (tc) TERT bound to a model RNA-DNA hybrid in the active site revealed the orientation of the 
RNA template with respect to the tcTRBD and supports the model that a high affinity RNA interaction in this region of TERT may establish the template boundary ${ }^{11}$. The first vertebrate TRBD structure was reported from the fugu fish Takifugu rubripes (tr), revealing the position of a conserved TFLY motif near the putative RNA binding region of trTRBD ${ }^{25}$. In this study, the TFLY motif was proposed to serve a similar role to the ciliate specific CP2-motif in promoting a high-affinity RNA interaction; however, the lack of RNA in the structure precluded a detailed description of the TRBD-RNA interaction network.

We set out to structurally determine the basis of template boundary definition in Tetrahymena telomerase. Here, we report the x-ray crystal structure of tTRBD bound to its cognate TBE RNA fragment. The structure highlights how the T-, CP-, and CP2-motifs within tTRBD cooperate to mediate interactions with the base of stem-loop II in Tetrahymena TER. The structure of tTRBD in complex with the TBE RNA explains previously reported biochemical mutagenesis experiments ${ }^{17,18,20-22}$, provides new insights into the mechanism of telomerase catalysis, and places novel constraints on the organization of the rest of the telomerase RNA subunit within the telomerase ribonucleoprotein (RNP) complex.

\section{RESULTS}

\section{Structure of TERT-RBD bound to the TBE RNA}

To determine the molecular mechanism of template boundary definition in Tetrahymena telomerase we sought to develop protein and RNA constructs amenable to high-resolution structure determination. The previously reported structure of the tTRBD does not include the cognate telomerase RNA or the ciliate specific CP2 peptide ${ }^{23}$. Prior mutagenesis experiments established the importance of the CP2-motif for protein-RNA interactions that direct template boundary definition ${ }^{20-22}$, and subsequent site-directed hydroxyl radical probing showed the CP2 peptide is in close spatial proximity to the TER TBE located at the base of stem-loop II ${ }^{21}$. Beginning with a tTRBD fragment (aa 195-516) that harbors multiple sites of RNA interaction including the CP2-motif ${ }^{14,21}$, we made a set of truncation mutants to identify a highly soluble tTRBD domain (aa 217-516) that retains sequences required for specific high-affinity interaction with TER (Fig. 1a).

We optimized the TER stem-loop II construct by replacing the native A22-A34 bulge with a canonical U22-A34 base pair (Fig 1b). This base substitution is observed naturally in several closely related ciliate TERs ${ }^{27}$, and does not impact Tetrahymena telomerase function when assayed in vitro and in vivo ${ }^{20,28}$. Indeed, full-length TER containing the A22U substitution supported wild type levels of binding to tTRBD (Supplementary Fig. 3a), as well as catalytic activity and template boundary definition (Supplementary Fig. 3b). To improve the folding efficiency of the isolated RNA stem construct, we separated stem-loop II into two short oligonucleotides (Fig 1b, inset). The tTRBD protein and the optimized TER-TBE RNA were mixed together at a 1:1.5 stoichiometry to yield a mono-dispersed protein-RNA complex as judged by size exclusion chromatography (Supplementary Fig. 3c). We crystallized the tTRBD-TBE complex and solved the structure to $3 \AA$ resolution by molecular replacement using the previously reported tTRBD structure as a search model (Table 1) ${ }^{23}$. The calculated electron density map permitted unambiguous modeling of most 
of the protein-RNA complex, with the exception of a small region of the tTRBD protein and several RNA nucleotides that are likely disordered in the crystal. The tTRBD-TBE complex crystallized in a 1:1 stoichiometry with two protein-RNA complexes (A and B) per asymmetric unit (Supplementary Fig. 4a). Complexes A and B demonstrated a high degree of overall similarity with an RMSD of $0.98 \AA^{2}$; however, regions of the protein-RNA binding interface in complex B were observed to be close to crystal packing contacts (Supplementary Fig. 4b and 4c.) and were substantially more disordered than in complex A, in which this same interface was far from any lattice contacts. We therefore focused our structural analyses on the more ordered complex A.

In the structure, the tTRBD is wedged into the base of the TER-TBE RNA stem II, diverting the paths of the 5'- and 3'-flanking strands in opposite directions (Fig. 2a). All protein-RNA contacts occur at the base of stem II, with the distal region of stem II (corresponding to the location of the native stem-loop II) extruding away from the protein interface. This arrangement is consistent with TER mutagenesis experiments and site-directed hydroxyl radical probing, which demonstrated that the base of stem II interacts with the tTRBD ${ }^{21}$, as well as with the observation that the distal region of stem-loop II is permissive to both sequence and structure modifications ${ }^{20,28}$. The three conserved sequence motifs within tTRBD (CP2, CP, and T) comprise the core RNA binding platform (Fig. 2a,b). Surprisingly, the majority of the protein-RNA contacts are not sequence specific with respect to TER, with a few notable exceptions (see below). Rather, the base of the TER-TBE is bound through an extensive network of polar contacts between the three conserved sequence motifs within TTRBD and the sugar-phosphate backbone of the RNA.

\section{The CP- and T-motifs position the CP2-motif for RNA binding}

The previously reported structure of the tTRBD (aa 254-519) revealed the organization of the conserved CP- and T-motifs ${ }^{23}$. Together, these regions of tTRBD form an electropositive groove that was hypothesized to represent a site of RNA interaction. However, our structure indicates that a major function of the T-CP pocket is to bind and orient the $\mathrm{CP} 2$ peptide (Fig. 3a), which is essential for RNA binding and was lacking in the previously crystallized construct ${ }^{23}$. The position of the CP2 peptide is fixed by a network of polar interactions between conserved amino acid side chains, as well as a number of backbone contacts. For example, the side chain of Arg226 in CP2 makes several hydrogen bonding interactions with the backbone of Phe408, Met411, Lys412 and Gln415, which together form a pocket that anchors the N-terminal region of the CP2 peptide within tTRBD (Fig. 3b). The hydroxyl group of Tyr231 in CP2 is hydrogen bonded to the guanidinium and carboxylate groups of Arg413 and Glu480, respectively (Fig. 3c). These polar contacts between Tyr231 and other regions of tTRBD induce a nearly $90^{\circ}$ kink in the protein backbone, redirecting the path of the CP2 peptide toward the base of the TBE RNA. The side chain of Arg473 in the T-motif makes hydrogen bonds with the backbone of CP2 residues Phe230 and Gln228 (Fig. 3d). In addition, the N1 position of the T-motif residue Trp496 indole group is hydrogen bonded with the carboxamide group of Asn233 in CP2. Finally, the terminal amino groups of two conserved lysines within the T-motif (Lys493 and Lys497) make polar backbone contacts with residues Cys 232 and Asn233 within the CP2 peptide (Fig. 3e). Thus, our structure of the tTRBD bound to the TBE RNA stem 
demonstrates that many of the conserved residues in the T-motif are indirectly required for RNA binding, and function to position the CP2 peptide in a geometry that is favorable for making RNA contacts.

\section{tTRBD-TBE interactions define the template boundary}

The TBE RNA stem forms an A-form helix (Fig. 4a), consistent with an earlier NMR structure of a model Tetrahymena stem-loop II sequence lacking the entire TBE region ${ }^{29}$. The crystal structure presented here reveals two critical RNA base pairs located at the interface between the TTRBD and the TBE RNA: a canonical Watson-Crick base pair between $\mathrm{C} 19$ and $\mathrm{G} 37$ and a previously unidentified wobble pair between U18 and U38 (Fig. 4b and Supplementary Fig. 5). The observation of these base pairing interactions and their central role in mediating RNA binding explains the high degree of sequence conservation observed for these nucleotide positions among ciliate telomerase RNAs ${ }^{27}$. Conserved residues from the $\mathrm{CP} 2-, \mathrm{CP}$-, and T- motifs all contribute to the tTRBD surface that binds the TBE RNA (Fig. 4c). Within the CP2 peptide, the guanidinium group of Arg237 extends into the major groove of the TBE RNA stem, making hydrogen bonding contacts with the Hoogsteen face of G37 and with the carbonyl group of U38, while the imidazole group of His234 makes a stabilizing polar contact to the carbonyl of U38 (Fig. $4 \mathrm{c}, \mathrm{d})$.

In addition to interactions with the helical portion of the TBE RNA, tTRBD makes a large number of polar contacts between the 5'-flanking single-stranded RNA and conserved Tand CP-motif side chains. Specifically, the Watson-Crick face of RNA nucleotide C15 makes hydrogen bonds with the side chains of Arg492 (T-motif) and Asn324 (CP-motif), and the 2' hydroxyl group of the $\mathrm{C} 15$ ribose moiety contacts the terminal amino group of Lys332 (CP-motif) (Fig. 4c,e). The side chains of several additional CP-motif residues (Lys328, Tyr337, Gln338, and Lys341) make polar contacts with the RNA phosphate backbone (Fig. 4c,e). With respect to the 3' single stranded RNA flanking the base of the TBE RNA stem, only a single nucleotide (C39) could be unambiguously fit into the electron density. Although nucleotides A40-U42 and U13 are distant from any lattice contacts, they are likely disordered in the crystal and therefore could not be fit into the electron density map. Several tTRBD residues make polar contacts with the phosphate backbone in the 3' single stranded region, including the guanidinium group of Arg473 (T-motif). Thus, our structure reveals the sequence and structure specific interactions between the CP2-,CP-, and $\mathrm{T}$-motif residues that mediate the protein-RNA interactions governing template boundary definition.

\section{Conservation and function of tTRBD-TBE interactions}

Many of the residues that participate in orienting the $\mathrm{CP} 2$ peptide, or that interact directly at the tTRBD-TBE binding interface (Fig. 5a,b), are highly conserved among ciliate TRBDs (Supplementary Fig. 2). The positions of these amino acids in our structure are in accord with this sequence conservation, as well as with the observation that alanine substitution at each of these sites can induce defects in RNA binding and/or telomerase activity 17,20-22. Notably, the two T-motif residues Glu480 and Arg492 are invariant among TRBDs from ciliates, yeasts, and vertebrates (Supplementary Fig. 1). The side chain of Arg492 in the T- 
motif makes base specific contacts with C15 in the TBE RNA (Fig. 5b), raising the possibility that this residue may be directly involved in RNA binding interactions in TRBDs from other organisms. A second invariant T-motif residue, Glu480, is hydrogen bonded to the side chain of Tyr231 in CP2 (Fig. 5a). Interestingly, the recently reported structure of the Takifugu rubripes TRBD identified the TFLY-motif, which is conserved among vertebrate TRBDs ${ }^{25}$. Comparison of the sequence of the CP2- and TFLY-motifs suggests that certain residues appear to be conserved (Fig. 5c). In order to ascertain whether the ciliate specific CP2-motif is structurally homologous to the vertebrate TFLY-motif, we aligned our structure with the two available vertebrate TRBD structures from Takifugu rubripes (tr) and the closely related Medaka fish Oryzias latipes (ol) (Fig. 5c). Indeed, in all three TRBD structures, the invariant T-motif glutamate side chain is well aligned, while Tyr321 from the olTRBD TFLY-motif is overlapping with Tyr231 from the tTRBD CP2-motif (Fig. 5d). In contrast, the corresponding tyrosine side chain from trTRBD (Tyr305) is significantly displaced. This alternative positioning of the TFLY-motif in the trTRBD structure is likely due to the fact that this region is directly involved in making crystal-packing contacts 25 . Further comparison of the tTRBD CP2-motif and the olTRBD TFLY-motif structures suggests additional regions of homology. For example, the sidechains of Phe 230 in tTRBD and Phe319 in olTRBD are in close proximity in the structural alignment. Lastly, two conserved basic tTRBD CP2 residues (His234 and Arg237) that comprise a sequence specific RNA binding patch are also present within olTRBD (Arg324 and His327), albeit with an inverted sequence order (Fig. 5e). Taken together, the results of our structural analysis suggest that the ciliate specific CP2-motif is structurally homologous to the vertebrate TFLY-motif, which may serve a similar function to mediate sequence specific protein-RNA interactions.

\section{A molecular model of template boundary definition}

In order to further understand the mechanism of template boundary definition in telomerase, we aligned the tTRBD-TBE structure with the complete TERT structure obtained from the flour beetle Tribolium castaneum bound to a model RNA-DNA hairpin in the active site ${ }^{11}$. The resulting model demonstrates the relative positions of the TBE and the template RNA within the telomerase complex (Fig. 6a). In this model, residue C39 is positioned $\sim 17 \AA$ from the nearest template RNA residue, roughly corresponding to the contour length of the three RNA nucleotides not present in the structural model. Previous work has demonstrated that the TBE is a site of protein-RNA interaction that is critical for preventing entry of nontemplate residues into the TERT active site ${ }^{20,21}$. The structural model we propose now directly demonstrates the mechanism by which this is accomplished. The location of the tTRBD-TBE complex is precisely positioned to prevent entry of non-template residue U42 into the active site of the enzyme, thereby establishing the template boundary.

\section{DISCUSSION}

We report the structure of the tTRBD in complex with the TBE of TER. The position of stem II with respect to TERT observed in our high-resolution structure is consistent with the proposed location of this same RNA element in a structural model derived from a recent 25 $\AA$ A cryo-EM Tetrahymena telomerase holoenzyme structure ${ }^{30}$. The most salient features of 
the tTRBD-TBE structure are the interactions between three conserved sequence motifs $(\mathrm{CP} 2, \mathrm{CP}$, and $\mathrm{T})$ and the manner in which these motifs cooperate to position the TBE RNA with respect to tTRBD. Previously reported biochemical experiments support a model in which a high-affinity interaction between TTRBD and the TBE RNA establishes template boundary definition by limiting the amount of RNA that may access the active site of the tTERT reverse transcriptase domain 17,18,20,21. More recent bulk biochemical and singlemolecule experiments demonstrated that the RNA template region of TER exists in either a compressed or extended conformation, depending on the stage of the telomere repeat synthesis reaction ${ }^{13}$. This finding supports the notion that the tTERT reverse transcriptase domain exerts a stretching force on the RNA template during telomere DNA synthesis, and that a point of protein-RNA contact must resist this pulling force to establish the template boundary. In our tTRBD structure bound to the TBE RNA, the protein domain is wedged against the base of the TBE RNA stem, making several sequence-specific contacts in the RNA major groove (Fig. 6a). This arrangement is well suited to provide mechanical stability against the reverse transcriptase pulling force (Fig. 6b), since both protein-RNA and RNARNA contacts would need to be disrupted to permit more RNA to enter the reverse transcriptase active site.

Further structural analysis is required to determine whether the mechanism of template boundary definition revealed by the TTRBD-TBE structure is a general feature found in telomerases from other organisms. The P1 stem found in vertebrate telomerase RNAs has been proposed to serve a functionally analogous role to Tetrahymena TER stem-loop II ${ }^{31}$. However, more recent experiments demonstrate that the sequence of the vertebrate telomerase RNA template can itself govern the location of the template boundary ${ }^{32}$. A recent study of the Takifugu rubripes TRBD structure identified a conserved protein motif (the TFLY-motif) present in vertebrate TERTs ${ }^{25}$. The TFLY-motif is positioned in the Takifugu TRBD structure nearby the position of the CP2-motif in the Tetrahymena TRBDTBE structure, indicating that the TFLY-motif may be the vertebrate homolog of the CP2motif in ciliates. An alignment between TRBD structures from Takifugu rubripes, the closely related Oryzias latipes ${ }^{26}$, and our Tetrahymena TRBD structure demonstrates a significant overlap in the position between the CP2-motif and the vertebrate TFLY-motifs (Fig. 5d,e). Indeed, Tyr231in the tTRBD structure is nearly identical in position to Tyr321 in the olTRBD, suggesting that the universally conserved tyrosine in the vertebrate TFLYmotif is the functional homolog of the tyrosine in the CP2-motif. This discovery should motivate future structural studies of vertebrate TRBDs bound to their cognate template proximal RNA fragments in order to determine the molecular basis of template boundary definition in vertebrates.

\section{ONLINE METHODS}

\section{RBD - TBE complex formation}

His-tagged RBD (217-516) was expressed in E.coli BL21-CodonPlus (DE3)-RP competent cells (Agilent) and purified by nickel exchange chromatography. RBD was further purified by size exclusion chromatography (Superdex 200 column). The TBE RNA construct was prepared by annealing two individual strands, each containing one side of Stem II (Fig. 1b). 
(Strand A: 5' UUCAUUCAGUUCU 3' Strand B: 5' UAGAACUGUCAUU 3'). To anneal the construct, the two strands were heated to $95^{\circ} \mathrm{C}$ for 3 minutes in annealing buffer (500 $\mathrm{mM} \mathrm{NaCl}, 20 \mathrm{mM}$ Tris- $\mathrm{HCl} \mathrm{pH} 7.8$ ) and then allowed to slowly cool to room temperature. After cooling to room temperature $\mathrm{MgCl}_{2}$ and DTT were each added to a final concentration of $1 \mathrm{mM}$. The tTRBD protein was mixed with the annealed TER-TBE RNA at a stoichiometry of $1: 1.5$ in high salt buffer $(500 \mathrm{mM} \mathrm{NaCl}, 20 \mathrm{mM}$ Tris- $\mathrm{HCl} \mathrm{pH} 7.8,1 \mathrm{mM}$ $\mathrm{MgCl}_{2}$ and $1 \mathrm{mM}$ DTT). This mixture was then dialyzed overnight ( 16 hours) into low salt buffer (100mM NaCl, 20mM Tris-HCl pH 7.8, $1 \mathrm{mM} \mathrm{MgCl} 2$ and $1 \mathrm{mM}$ DTT). Formation of protein-RNA complex was analyzed using diagnostic sizing chromatography on a Superdex 200 column (Supplemental Fig. 3c) prior to setting crystallization trays.

\section{Protein crystallization and data collection}

tTRBD-TBE RNA crystals that diffracted to $3 \AA$ appeared overnight and grew to final size of $\sim 10-20$ microns after 4-5 days. Crystals were grown by the sitting-drop vapor-diffusion method using the ARI GRYPHON in Hampton Research Natrix HT condition A5 (200mM $\mathrm{KCl}, 10 \mathrm{mM} \mathrm{MgCl} 2,50 \mathrm{mM}$ MES monohydrate $\mathrm{pH} 5.6,4 \%$ PEG 8000) at a 3:1 ratio of sample to screen. Crystals were transferred into cryoprotectant solution consisting of $20 \%$ ethylene glycol, $160 \mathrm{mM} \mathrm{KCl}, 8 \mathrm{mM} \mathrm{MgCl} 2,40 \mathrm{mM}$ MES monohydrate $\mathrm{pH}$ 5.6, 3.2\% PEG 8000 at $4^{\circ} \mathrm{C}$. Crystals were harvested by flash freezing in liquid nitrogen. X-ray data was collected at the ALS beamline 501 at a wavelength of $1 \AA$ and a temperature of $100 \mathrm{~K}$. The data was processed using MOSFILM. Crystals are in the P212121 space group and there are two tTRBD-TBE complexes in the asymmetric unit.

\section{Structure determination and refinement}

Phases were calculated in CCP4 by molecular replacement using the previously solved Tetrahymena RBD structure (PDB 2R4G ${ }^{23}$ ) as the initial search model.. Model building was performed in Coot and refined using PHENIX. The structure was refined to good stereochemistry with $93 \%$ of residues in the most favored region, $6 \%$ in the allowed region and $1 \%$ outliers of the Ramachandran plot.

\section{Supplementary Methods}

\section{Electrophoretic Mobility Shift Assay (EMSA)}

WT TER and TER A22U were transcribed in vitro using T7 RNA polymerase and PCR templates. The RNA was gel purified, CIP and DNase treated and ethanol precipitated. The RNA was then end-labeled with radiolabeled ATP. RNA was heated to $60^{\circ} \mathrm{C}$ and let cool to room temperature on bench. RDB was diluted into binding buffer containing $10 \%$ glycerol, $20 \mathrm{mM}$ Tris $\mathrm{pH} 8.0,100 \mathrm{mM} \mathrm{NaCl}, 1 \mathrm{mM} \mathrm{MgCl} 2$ and $1 \mathrm{mM}$ DTT. Each binding reaction contained the indicated amount of tTRBD plus $0.4 \mathrm{nM}$ end-labeled RNA in binding buffer containing $10 \%$ glycerol, $20 \mathrm{mM}$ Tris $\mathrm{pH} 8.0,100 \mathrm{mM} \mathrm{NaCl}, 1 \mathrm{mM} \mathrm{MgCl}_{2}, 1 \mathrm{mM}$ DTT, $0.1 \mathrm{mg} / \mathrm{ml} \mathrm{BSA}, 0.1 \mathrm{mg} / \mathrm{ml}$ yeast tRNA and $0.1 \mathrm{ul}$ RNasin. Reactions were incubated for 20 min at $30^{\circ} \mathrm{C}$. Samples were run on a $5 \%$ native acrylamide gel (37.5:1 acrylamide: bis acrylamide, $4 \%$ glycerol, $0.5 \times \mathrm{TBE}$ ) and run for $3 \mathrm{~h}$ at $4^{\circ} \mathrm{C}$ at $200 \mathrm{~V}$. The gel was dried and imaged overnight followed by PhosphorImager analysis. 


\section{Primer extension assay}

Telomerase for in vitro primer extension assays was prepared in RRL (Promega) as described previously ${ }^{13} .2 \mu \mathrm{L}$ of the RRL telomerase reconstitution reaction was added to 2 $\mu \mathrm{M}$ DNA primer (GGGGTT) $)_{3}, 100 \mu \mathrm{M}$ dTTP, $9 \mu \mathrm{M}$ dGTP, $1 \mu \mathrm{M}{ }^{32} \mathrm{P}$ a-dGTP, in a final volume of $15 \mu \mathrm{L}$ in a buffer containing $50 \mathrm{mM}$ Tris $\mathrm{pH} 8.0,1.25 \mathrm{mM} \mathrm{MgCl}_{2}$, and $10 \%$ glycerol. To test for template boundary defects, reactions were performed in the presence or absence $100 \mu \mathrm{M}$ dATP. Reactions were incubated for 1 hour at $30^{\circ} \mathrm{C}$ and then phenol:chloroform extacted and ethanol precipitated. Extension products were resolved on a $12 \%$ PAGE DNA sequencing gel and imaged using a Typhoon scanner with a phosphor imaging screen.

\section{Supplementary Material}

Refer to Web version on PubMed Central for supplementary material.

\section{Acknowledgments}

We thank the laboratory of K. Collins at University of California Berkeley for the original tTRBD expression plasmids. We thank L. Lancaster and S. Tripathy for technical help with crystallization and data analysis. We thank H. Noller, W. Scott, and members of S. Rubin's laboratory for helpful discussions and technical advice. This work was supported by grants from the US National Institute of Health (2T32GM008646-16 to L.I.J) and (RO1GM095850 to M.D.S.)

\section{REFERENCES}

1. Palm W, de Lange T. How shelterin protects mammalian telomeres. Annu Rev Genet. 2008; 42:301-34. [PubMed: 18680434]

2. Allsopp RC, et al. Telomere length predicts replicative capacity of human fibroblasts. Proc Natl Acad Sci U S A. 1992; 89:10114-8. [PubMed: 1438199]

3. Greider CW, Blackburn EH. The telomere terminal transferase of Tetrahymena is a ribonucleoprotein enzyme with two kinds of primer specificity. Cell. 1987; 51:887-98. [PubMed: 3319189]

4. Vulliamy TJ, Dokal I. Dyskeratosis congenita: the diverse clinical presentation of mutations in the telomerase complex. Biochimie. 2008; 90:122-30. [PubMed: 17825470]

5. Kim NW, et al. Specific association of human telomerase activity with immortal cells and cancer. Science. 1994; 266:2011-5. [PubMed: 7605428]

6. Licht JD, Collins K. Telomerase RNA function in recombinant Tetrahymena telomerase. Genes Dev. 1999; 13:1116-25. [PubMed: 10323863]

7. Greider CW, Blackburn EH. A telomeric sequence in the RNA of Tetrahymena telomerase required for telomere repeat synthesis. Nature. 1989; 337:331-7. [PubMed: 2463488]

8. Lue NF. A physical and functional constituent of telomerase anchor site. J Biol Chem. 2005; 280:26586-91. [PubMed: 15905172]

9. Romi E, et al. High-resolution physical and functional mapping of the template adjacent DNA binding site in catalytically active telomerase. Proc Natl Acad Sci U S A. 2007; 104:8791-6. [PubMed: 17494734]

10. Zaug AJ, Podell ER, Cech TR. Mutation in TERT separates processivity from anchor-site function. Nat Struct Mol Biol. 2008; 15:870-2. [PubMed: 18641663]

11. Mitchell M, Gillis A, Futahashi M, Fujiwara H, Skordalakes E. Structural basis for telomerase catalytic subunit TERT binding to RNA template and telomeric DNA. Nat Struct Mol Biol. 2010; 17:513-8. [PubMed: 20357774]

12. Parks JW, Stone MD. Coordinated DNA dynamics during the human telomerase catalytic cycle. Nat Commun. 2014; 5:4146. [PubMed: 24923681] 
13. Berman AJ, Akiyama BM, Stone MD, Cech TR. The RNA accordion model for template positioning by telomerase RNA during telomeric DNA synthesis. Nat Struct Mol Biol. 2011; 18:1371-5. [PubMed: 22101935]

14. O'Connor CM, Lai CK, Collins K. Two purified domains of telomerase reverse transcriptase reconstitute sequence-specific interactions with RNA. J Biol Chem. 2005; 280:17533-9. [PubMed: 15731105]

15. Jacobs SA, Podell ER, Cech TR. Crystal structure of the essential N-terminal domain of telomerase reverse transcriptase. Nat Struct Mol Biol. 2006; 13:218-25. [PubMed: 16462747]

16. Akiyama BM, Parks JW, Stone MD. The telomerase essential N-terminal domain promotes DNA synthesis by stabilizing short RNA-DNA hybrids. Nucleic Acids Res. 2015

17. Bryan TM, Goodrich KJ, Cech TR. Telomerase RNA bound by protein motifs specific to telomerase reverse transcriptase. Mol Cell. 2000; 6:493-9. [PubMed: 10983995]

18. Lai CK, Mitchell JR, Collins K. RNA binding domain of telomerase reverse transcriptase. Mol Cell Biol. 2001; 21:990-1000. [PubMed: 11158287]

19. Theimer CA, Feigon J. Structure and function of telomerase RNA. Curr Opin Struct Biol. 2006; 16:307-18. [PubMed: 16713250]

20. Lai CK, Miller MC, Collins K. Template boundary definition in Tetrahymena telomerase. Genes Dev. 2002; 16:415-20. [PubMed: 11850404]

21. Akiyama BM, Gomez A, Stone MD. A conserved motif in Tetrahymena thermophila telomerase reverse transcriptase is proximal to the RNA template and is essential for boundary definition. $\mathrm{J}$ Biol Chem. 2013; 288:22141-9. [PubMed: 23760279]

22. Miller MC, Liu JK, Collins K. Template definition by Tetrahymena telomerase reverse transcriptase. EMBO J. 2000; 19:4412-22. [PubMed: 10944124]

23. Rouda S, Skordalakes E. Structure of the RNA-binding domain of telomerase: implications for RNA recognition and binding. Structure. 2007; 15:1403-12. [PubMed: 17997966]

24. Gillis AJ, Schuller AP, Skordalakes E. Structure of the Tribolium castaneum telomerase catalytic subunit TERT. Nature. 2008; 455:633-7. [PubMed: 18758444]

25. Harkisheimer M, Mason M, Shuvaeva E, Skordalakes E. A motif in the vertebrate telomerase Nterminal linker of TERT contributes to RNA binding and telomerase activity and processivity. Structure. 2013; 21:1870-8. [PubMed: 24055314]

26. Huang J, et al. Structural basis for protein-RNA recognition in telomerase. Nat Struct Mol Biol. 2014; 21:507-12. [PubMed: 24793650]

27. McCormick-Graham M, Romero DP. Ciliate telomerase RNA structural features. Nucleic Acids Res. 1995; 23:1091-7. [PubMed: 7739888]

28. Cunningham DD, Collins K. Biological and biochemical functions of RNA in the tetrahymena telomerase holoenzyme. Mol Cell Biol. 2005; 25:4442-54. [PubMed: 15899850]

29. Richards RJ, Theimer CA, Finger LD, Feigon J. Structure of the Tetrahymena thermophila telomerase RNA helix II template boundary element. Nucleic Acids Res. 2006; 34:816-25. [PubMed: 16452301]

30. Jiang J, et al. The architecture of Tetrahymena telomerase holoenzyme. Nature. 2013; 496:187-92. [PubMed: 23552895]

31. Chen JL, Greider CW. Template boundary definition in mammalian telomerase. Genes Dev. 2003; 17:2747-52. [PubMed: 14630939]

32. Brown AF, et al. A self-regulating template in human telomerase. Proc Natl Acad Sci U S A. 2014; 111:11311-6. [PubMed: 24982163] 
a

Telomerase Reverse Transcriptase (TERT)

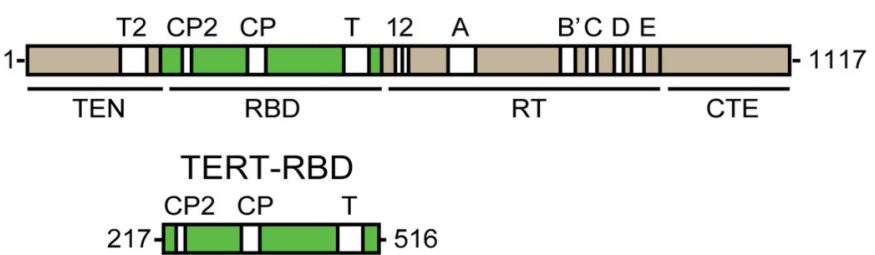

b

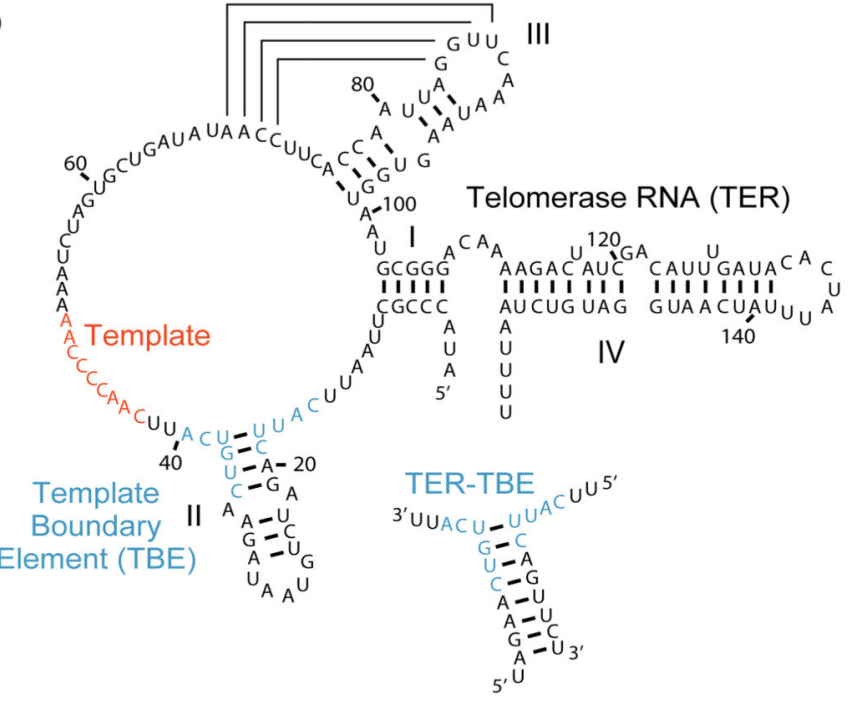

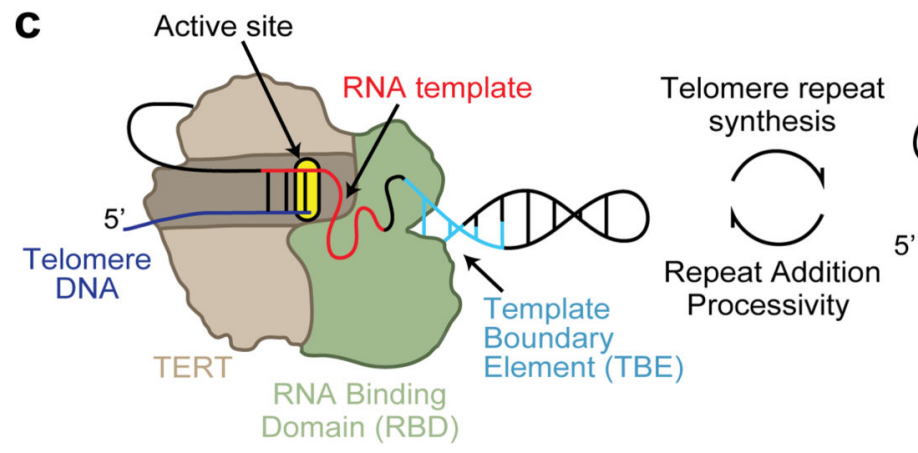

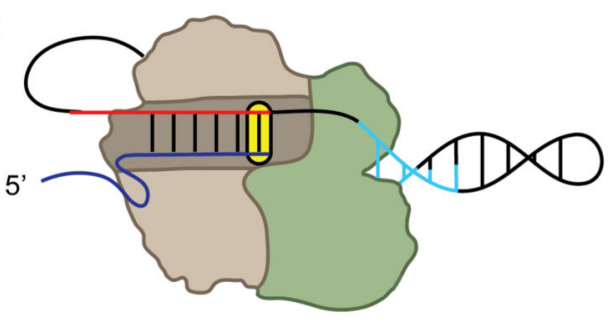

Figure 1.

Tetrahymena thermophila telomerase composition and catalytic cycle.

(a) Primary structure of the T. thermophila telomerase reverse transcriptase (TERT). The TEN, RT and CTE domains are shown in brown and the RNA binding domain (RBD) is shown in green. Conserved sequence motifs are shown as white boxes. The RBD construct used in this study is shown in green under the full-length TERT. (b) Secondary structure model of the T. thermophila telomerase RNA (TER). The RNA template is shown in red and the template boundary element (TBE) is shown in cyan (inset). The modified TBE RNA construct used in the present study. (c) Model for T. thermophila telomerase catalysis with the TERT RT domain in brown, RBD domain in green, the template RNA in red and the telomere DNA in blue. 


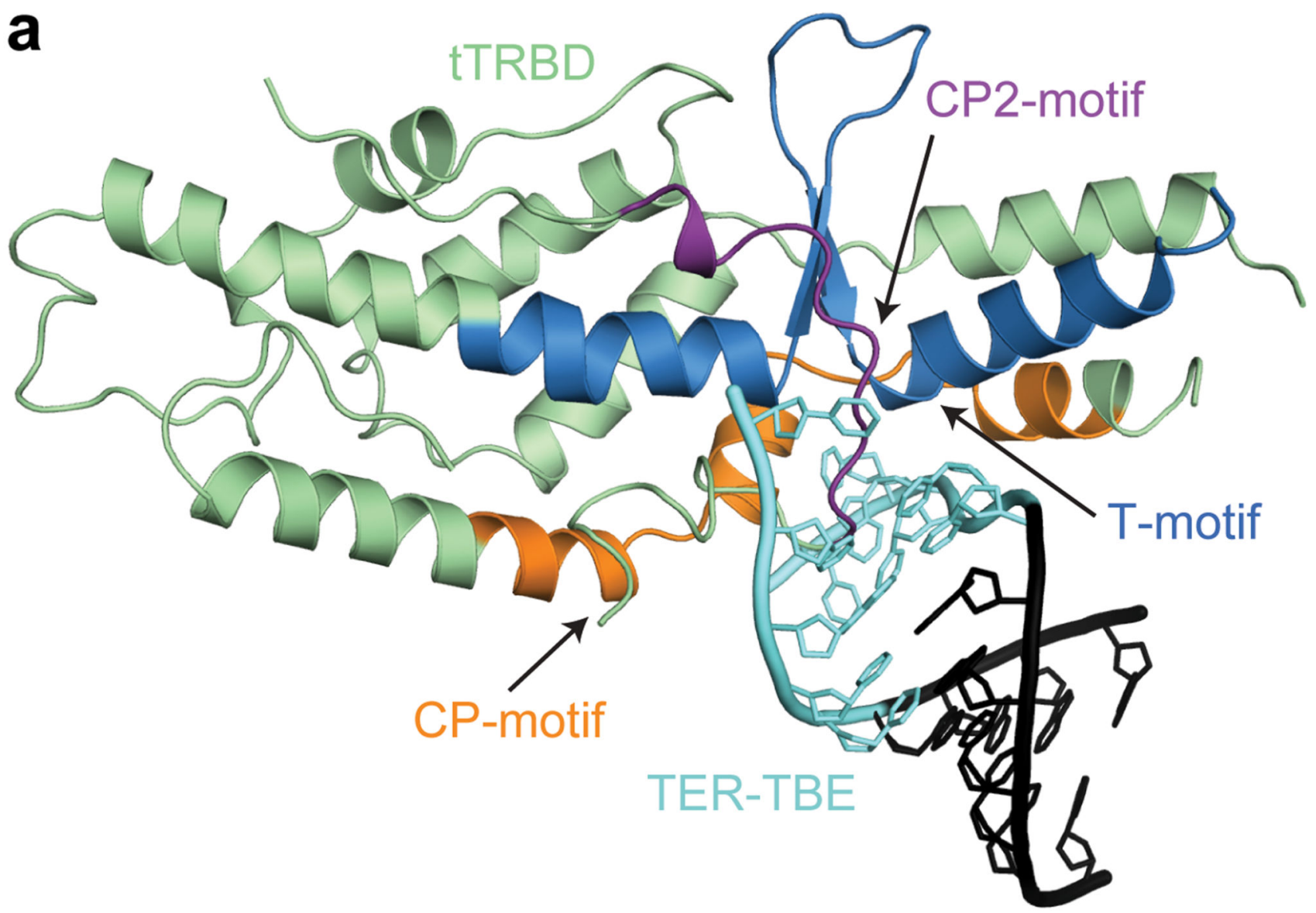

b

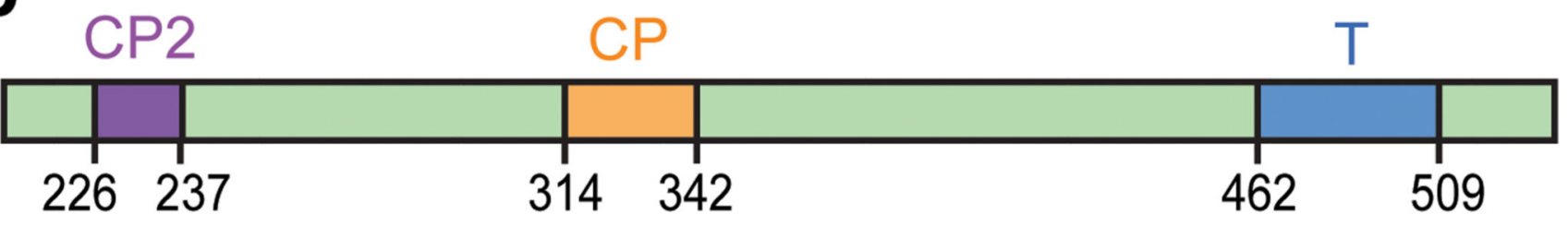

Figure 2.

Structure of the tTRBD-TBE complex.

(a) Cartoon representation of the tTRBD-TBE complex. tTRBD is shown in light green with the CP2-, CP-, and T-motifs shown in purple, orange and blue, respectively. The TBE is shown in cyan and the remaining stem II RNA in black. (b) Primary structure organization of tTRBD. Coloring scheme for each of the conserved sequence motifs is as described in a. 

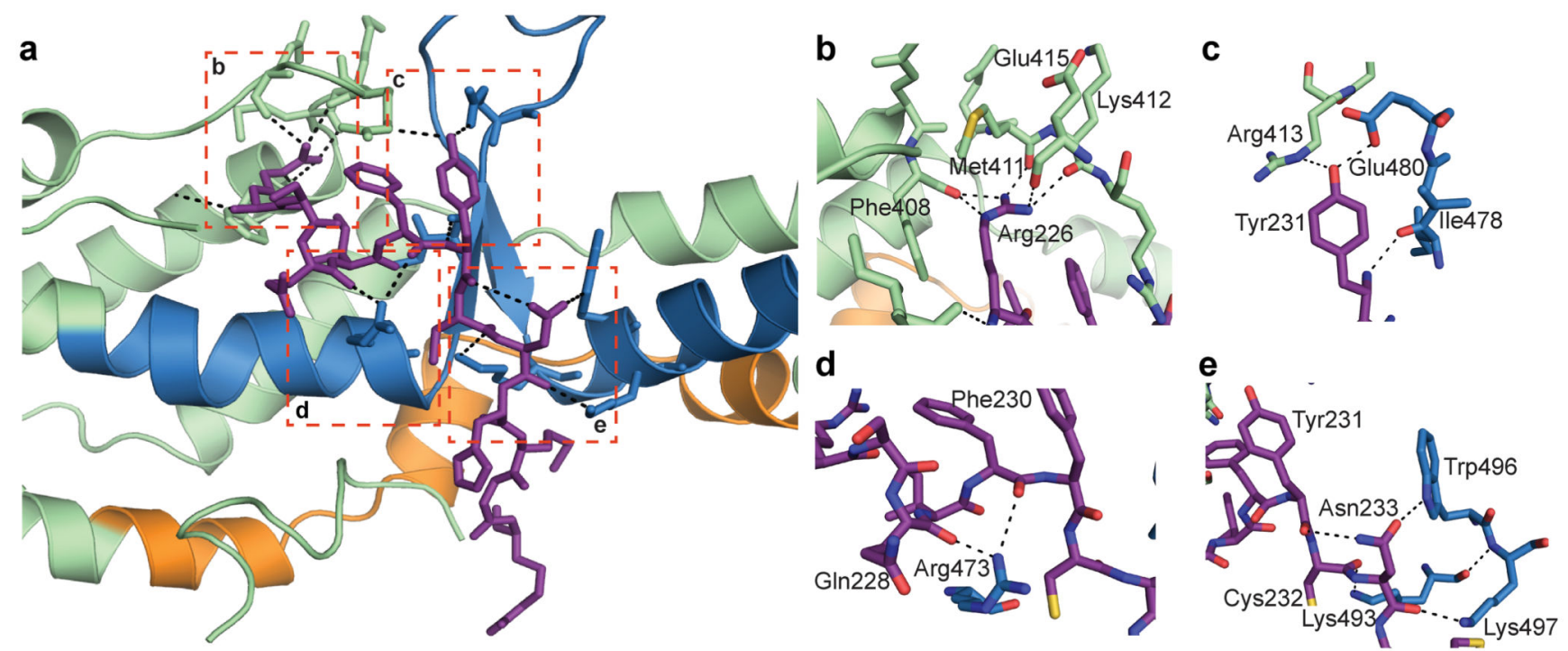

Figure 3.

Protein-protein interactions between conserved tTERT RBD motifs.

(a) Cartoon representation highlighting $\mathrm{CP} 2$ peptide (purple) interactions. Coloring scheme is same as in Fig. 2a. Interactions outlined by red dashed boxes are highlighted in panels b-e. (b) Arg226 makes polar contacts with the core of RBD. (c) Tyr231 interacts with the Tmotif residue Glu480. (d) Gln228 and Phe230 make polar contacts with the T-motif residue Arg473. (e) Asn233 interacts with T-motif residues Trp496 and Lys497, as well as with CP2 peptide residue Tyr321. 


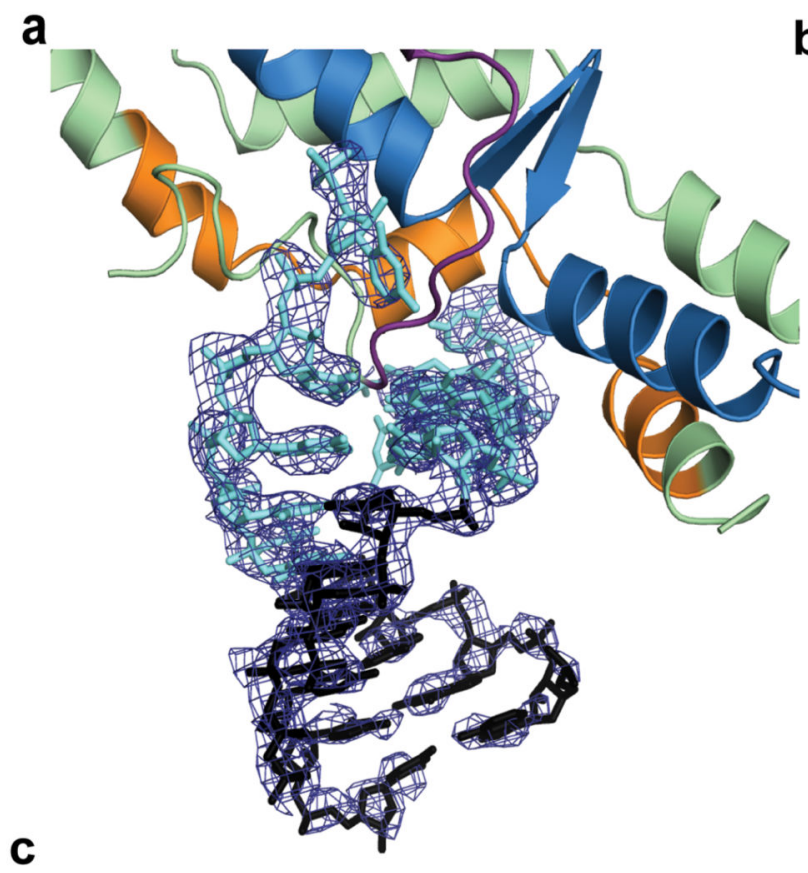

b

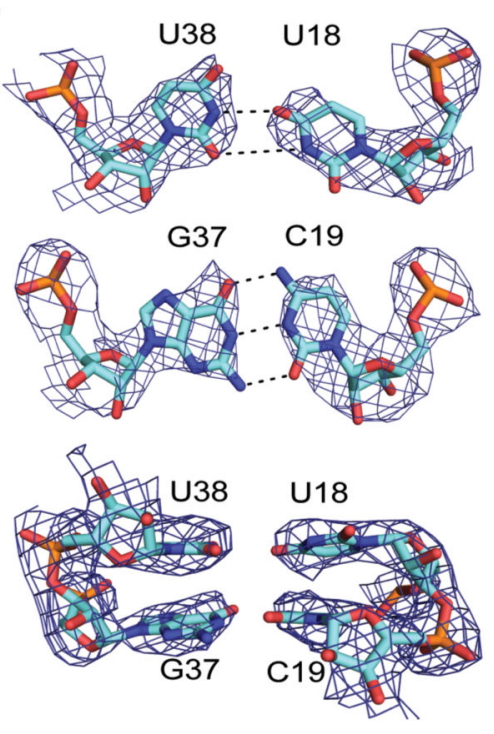

d
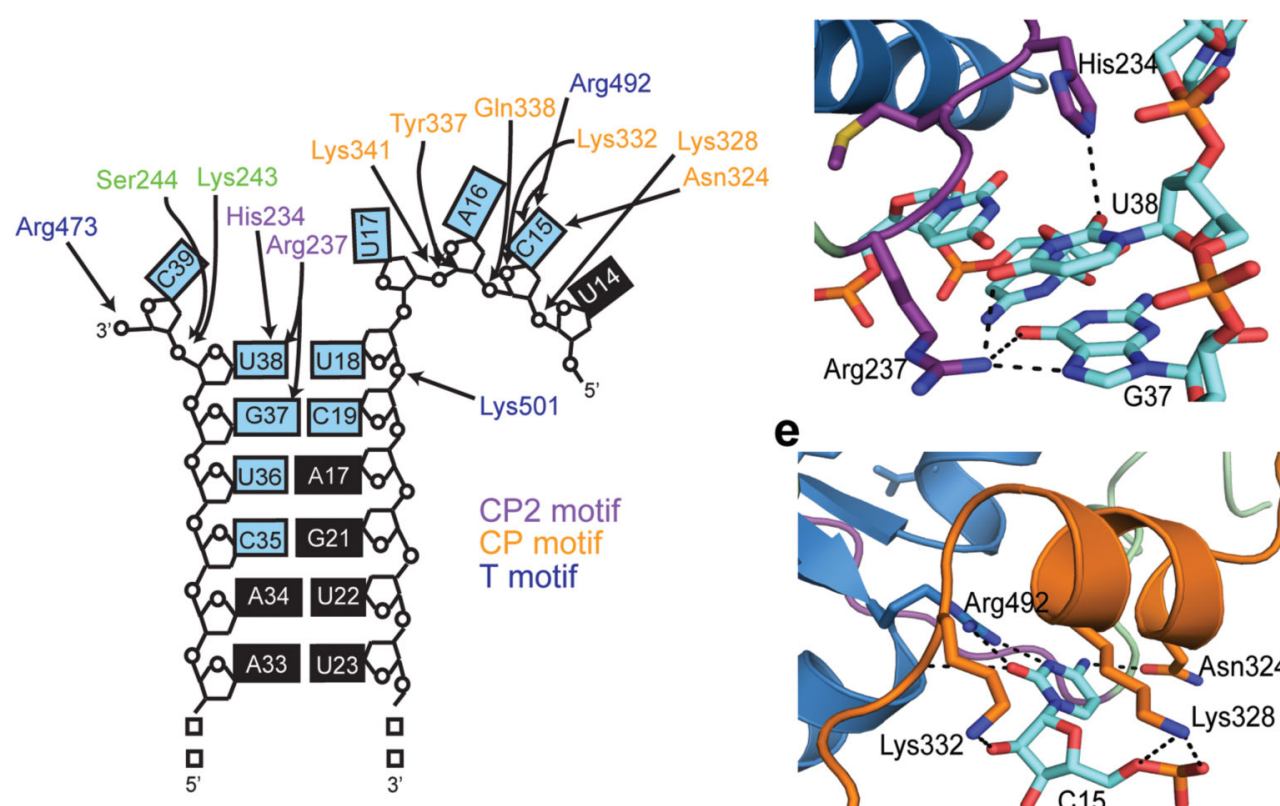

e

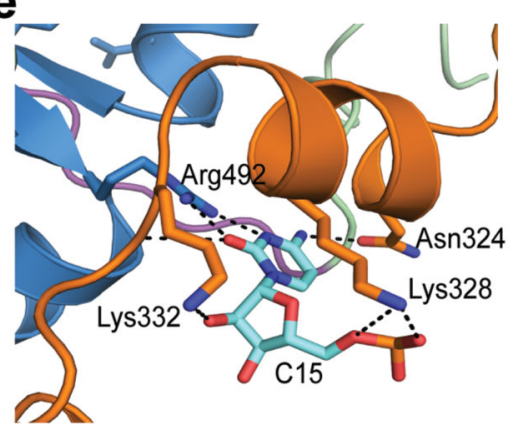

Figure 4.

Protein-RNA interactions within the tTRBD-TBE complex. (a) Simulated annealing omit map of the TER-TBE RNA at $2.0 \sigma$ contour level superimposed on cartoon model of tTRBDTBE structure. (b) Simulated annealing omit map of the U38-U18 and G37-C19 base pairs at contoured to $1.5 \sigma$. The bottom panel shows a side view of the stacked U23-U18 and G37C19 base pairs. (c) Schematic diagram of tTRBD-TER TBE interactions. The TBE RNA nucleotides are colored in cyan and the rest of the RNA in black. tTRBD residues from CP2-, CP-, and T-motif residues are shown in purple, orange and blue, respectively. Arrows 
indicate sites of polar interactions. (d) Arg237 interacts with nucleotides U38 and G37 at the base of the TBE RNA stem. His234 also interacts with U38. (e) The TBE RNA nucleotide $\mathrm{C} 15$ fits into a polar pocket between the CP- and T-motifs. C15 makes polar contacts with T-motif residue Arg492 and CP-motif residue Asn324, Lys332 and Lys328. 
a

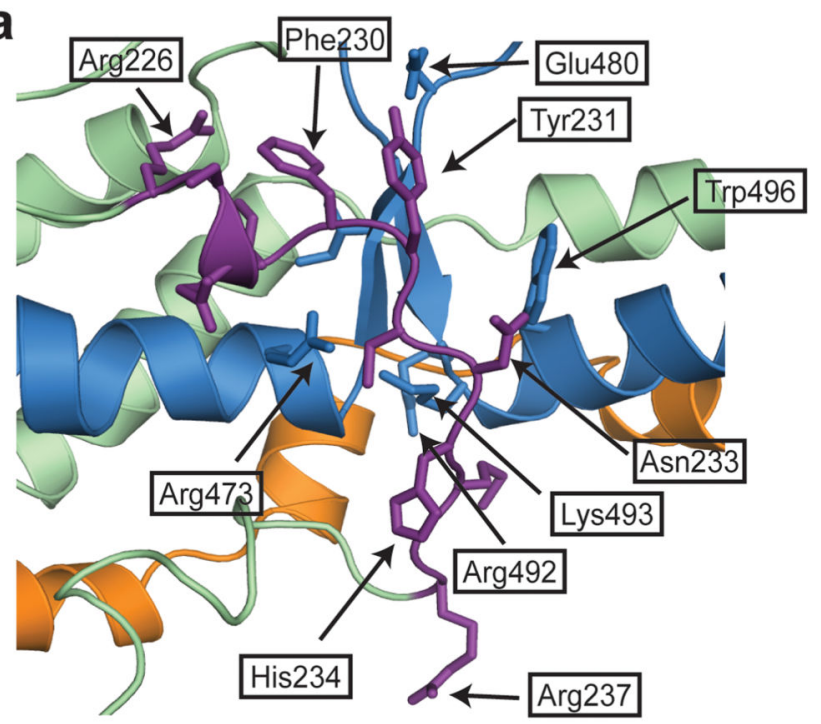

b

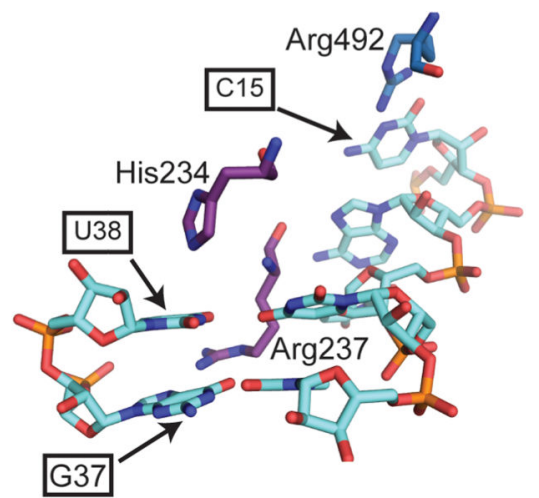

C

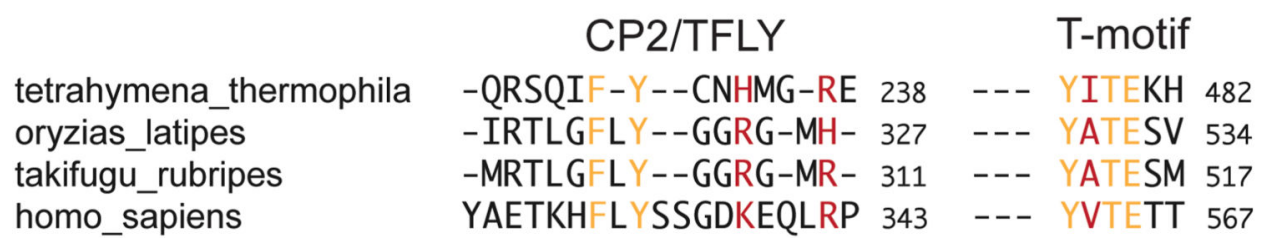

d

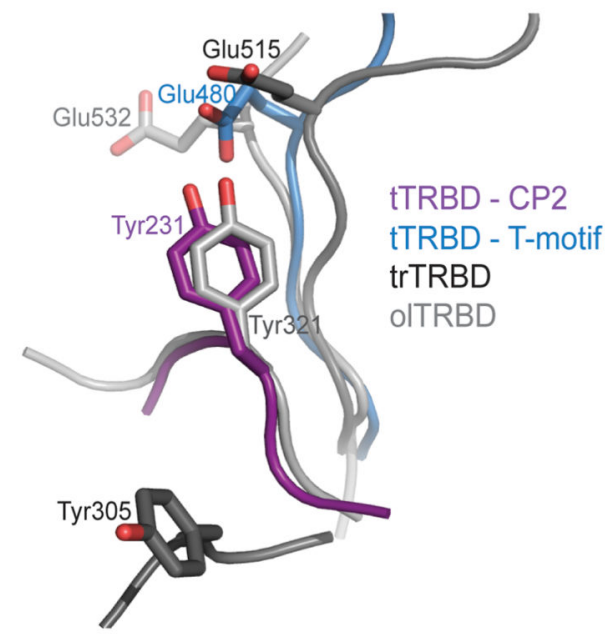

e

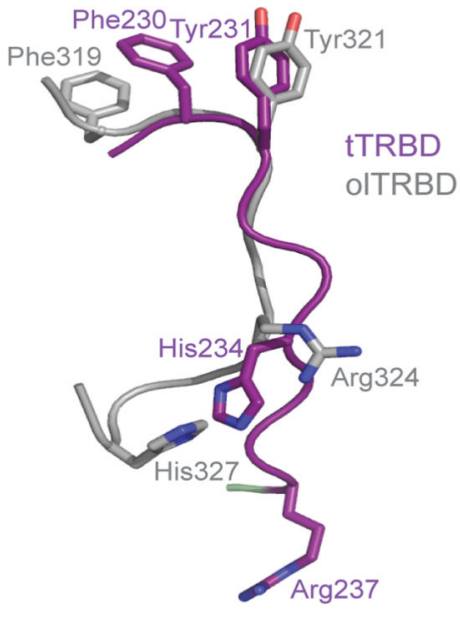

Figure 5.

Conservation and function of observed interactions in the tTRBD-TBE complex. (a) Conserved residues in the $\mathrm{CP} 2$ - and T-motifs that are important for RNA binding and/or telomerase function. Boxed residues represent $\mathrm{CP} 2$ residues that are conserved across ciliates and T-motif residues that are conserved across species. (b) Conserved RNA residues that are critical for RBD binding. Boxed nucleotides represent nucleotides that are conserved across ciliates and that have previously been demonstrated to be important for RBD binding. (c) CP2/TFLY alignment. The CP2 motif of Tetrahymena was aligned manually to the 
TFLY motif of vertebrates. Invariant residues are shown in orange and residues with conserved chemical properties are shown in red. A segment of the conserved T-motif alignment is also shown. (d) Structural alignment of tTRBD, trTRBD and olTRBD. tTRBD CP2-motif and T-motif are shown in purple and blue, respectively. trTRBD and olTRBD are dark grey and light grey, respectively (PDB 4LMO ${ }^{25}$ ) (PDB 4O26 ${ }^{26}$ ). (e) Structural alignment of the tTRBD CP2 motif and the olTRBD TFLY motif. Color scheme is same as in d. 

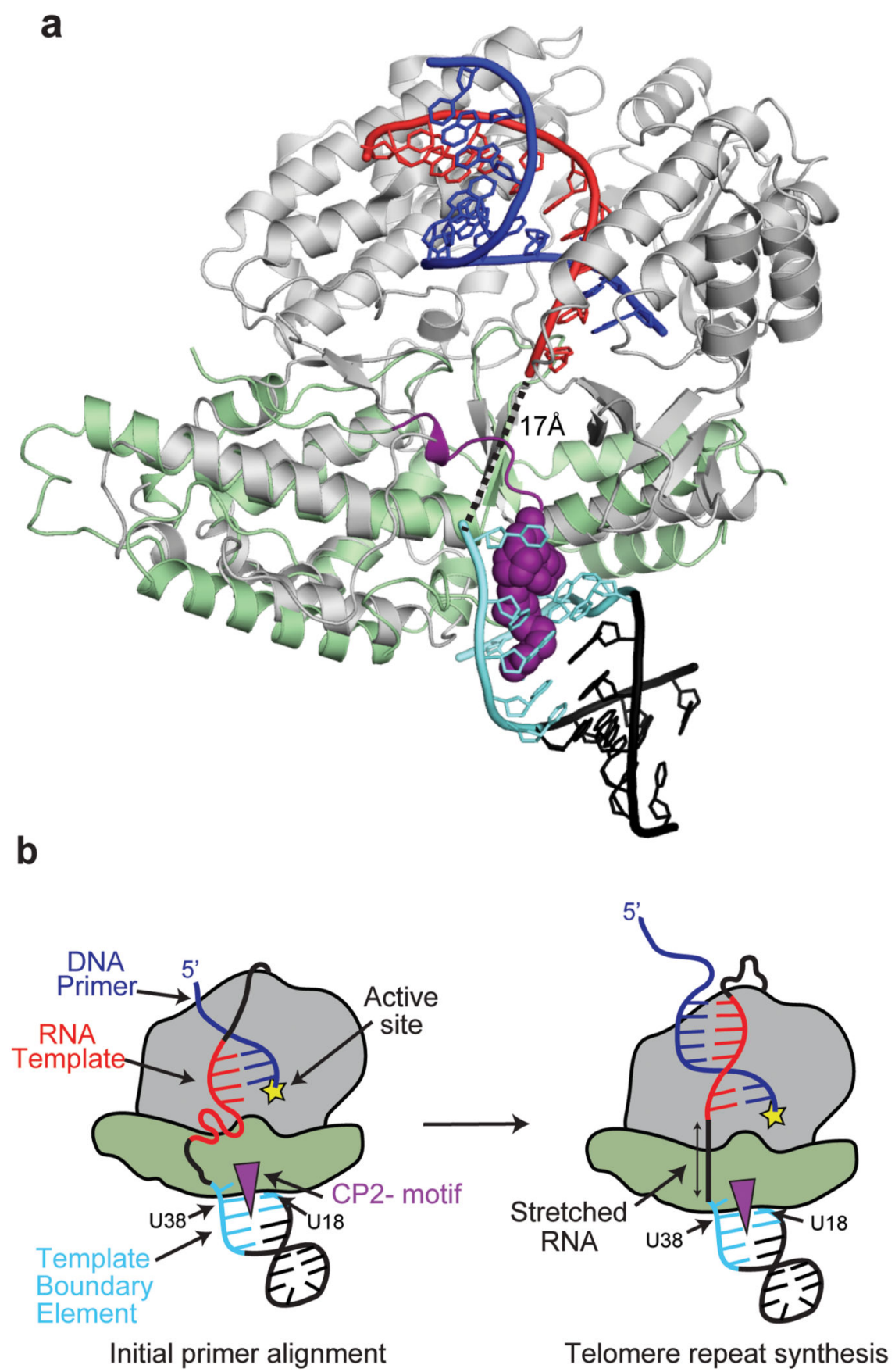

Figure 6.

Structural model of $T$. thermophila telomerase RNA template connectivity to the TER template boundary element. (a) Structure of the tTRBD (light green) bound to the TER-TBE RNA (cyan and black) aligned to the $T$. castaneum TERT structure (grey) bound to a model RNA template (red) and DNA primer (blue) (PDB 3KYL ${ }^{11}$ ). Dashed line indicates distance ( 17 A) between the 3' terminus of the TER-TBE and the 5' terminus of the RNA template in model. (b) Cartoon model for template boundary definition in Tetrahymena telomerase. The tTRBD is shown in green, the TERT active site as a yellow star, the DNA primer in 
blue, the RNA template in red, the TBE RNA in cyan, and the CP2 peptide as a purple triangle. 
Table 1

Data collection and refinement statistics (molecular replacement)

\begin{tabular}{|c|c|}
\hline & RBD-TBE \\
\hline \multicolumn{2}{|l|}{ Data collection } \\
\hline Space group & P212121 \\
\hline \multicolumn{2}{|l|}{ Cell dimensions } \\
\hline$a, b, c(\AA)$ & $\begin{array}{l}58.53,117.77 \\
131.18\end{array}$ \\
\hline$a, \beta, \gamma\left(^{\circ}\right)$ & $90.00,90.00,90.00$ \\
\hline Resolution ( $(̊)$ & $41.51(3.00) *$ \\
\hline$R_{\text {merge }}$ & $0.231(0.969)$ \\
\hline$I / \sigma I$ & $7.8(2.1)$ \\
\hline Completeness (\%) & $99.5(99.3)$ \\
\hline $\mathrm{CC}(1 / 2)$ & $0.990(0.720)$ \\
\hline Redundancy & $6.8(6.8)$ \\
\hline \multicolumn{2}{|l|}{ Refinement } \\
\hline Resolution ( $(\AA)$ & $41.51-3.00$ \\
\hline No. reflections & 18649 \\
\hline$R_{\text {work }} / R_{\text {free }}$ & $0.239 / 0.290$ \\
\hline No. atoms & 4793 \\
\hline Protein & 4767 \\
\hline Ligand/ion & 2 \\
\hline Water & 24 \\
\hline \multicolumn{2}{|l|}{$B$-factors } \\
\hline Protein & 62.90 \\
\hline Ion & 65.50 \\
\hline Water & 43.80 \\
\hline \multicolumn{2}{|l|}{ R.m.s. deviations } \\
\hline Bond lengths (Å) & 0.003 \\
\hline Bond angles $\left({ }^{\circ}\right)$ & 0.82 \\
\hline
\end{tabular}

